\title{
Laparoscopic Antireflux Surgery: A Comparative Study of Nissen Fundoplication and Hiatoplasty With and Without Absorbable Biological Mesh
}

\author{
Zackariah Clement $^{\mathrm{a}, \mathrm{c}}$, Phil Jeans ${ }^{\mathrm{b}}$
}

\begin{abstract}
Background: Laparoscopic Nissen fundoplication with hiatoplasty has been shown to be an effective antireflux operation. The use of biological mesh in hiatoplasty has been controversial.

Method: This retrospective comparative study included all patients with severe symptomatic gastro-oesophageal reflux disease who underwent antireflux surgery with and without biological mesh repair in one surgeons practice, 202 patients who underwent Nissen fundoplication with hiatus hernia repair between 2000 and 2010 were retrospectively analysed. Patients were divided into 4 groups: large paraoesophageal hernia with Surgisis mesh $(n=24)$ and without $(n=61)$; and patients without hiatal hernia with Surgisis mesh $(n=26)$ and without $(n=91)$. The Incidence of recurrence of hiatal hernia and post-operative side-effects were the outcome measures.
\end{abstract}

Results: In the group with paraoesophageal hernia at approximately 6 months postoperatively, the side-effects reported by patients without Surgisis mesh were reflux (15\%), flatulence (28\%), dysphagia $(46 \%)$, and hiatal hernia recurrence $(13 \%)$. Comparatively, patients receiving Surgisis mesh repair reported side effects of reflux (8\%), flatulence $(17 \%)$, dysphagia $(75 \%)$, and hiatal hernia recurrence (4\%). In the group without large hernia, the side-effects reported by patients without Surgisis mesh were reflux (12\%), flatulence $(20 \%)$, dysphagia $(41 \%)$, and incidence of hiatal hernia $(1 \%)$ and in patients with Surgisis mesh repair the rates of side-effects were: reflux $(11 \%)$, flatulence $(19 \%)$, dysphagia (69\%), and incidence of hiatal hernia (3.8\%).

Conclusion: This retrospective analysis shows that antireflux sur-

Manuscript accepted for publication May 24, 2013

\footnotetext{
${ }^{\mathrm{a}}$ General Surgery Registrar, the Campbelltown Hospital, Sydney, Therry Road, Campbelltown, NSW 2560, Australia

${ }^{b}$ Upper Gastrointestinal and Hepatobiliary Surgeon, the Canberra Hospital, Canberra, Yamba Drive, Garran, ACT 2605, Australia ${ }^{\mathrm{c} C}$ Corresponding author: Zackariah Clement, General Surgery Registrar, the Campbelltown Hospital, Sydney, Therry Road, Campbelltown, NSW 2560, Australia. Email: zackariahc@yahoo.com
}

gery with an absorbable biological mesh hiatoplasty is safe, and may lead to a reduction in the incidence of recurrent hiatal hernia. It also produces good control of reflux, with low risk of side-effects and complications.

Keywords: Nissen fundoplication; Biological mesh; Antireflux surgery

\section{Introduction}

Anti-reflux surgery has been evolving for over half a century. Surgical management is reserved for patients with complications of reflux such as recurrent or refractory oesophagitis, stricture, Barrett's metaplasia, and persisting reflux symptoms despite medical management. Laparoscopic Nissen fundoplication has been an established, effective and safe treatment option for patients with GORD with or without hiatus hernia [1-4].

One of the most frequent anatomical and technical failures in anti-reflux surgery is recurrent hiatal hernia and wrap migration. Successful repair of the hiatus hernia depends on the integrity of the tissue and the tension needed to approximate it. Any closure method is prone to disruption since the diaphragm is under repetitive stress due to the mechanics of respiration $[5,6]$. Hence there has been an increasing vogue for using prosthetic material to reinforce the repair in large hiatus hernia, particularly since the recurrence rate following this type of surgery is high, and recurrent hiatal herniation accounts for up to $70 \%$ of re-operations for failed anti-reflux surgery $[3,5,7]$. The use of biological mesh during hiatal hernia repair has been shown to decrease the recurrence rate of hernia lower than that obtained historically without the mesh [8]. The use of synthetic mesh is associated with complications such as oesophageal stricture, erosion, perforation, migration of mesh into oesophagus or stomach, and infections [9-11]. This has led to debate regarding the type of mesh material, size, shape, and fixation techniques. The biological porcine collagen mesh has been shown to reduce complications and recurrence [8].

Porcine collagen mesh (Surgisis) is an acellular xeno- 
Table 1. Post-Operative Side Effects in LHH Group

Patients with large hiatus hernia (mean 6 months follow-up)

\begin{tabular}{|c|c|c|c|c|c|}
\hline$N=85$ & Reflux & Flatulence & Dysphagia & Post-op endoscopy & Recurrence \\
\hline No mesh $(\mathrm{N}=61)$ & 9 & 17 & 29 & 22 & $8(13 \%)$ \\
\hline Surgisis mesh $(\mathrm{N}=24)$ & 7 & 4 & 15 & 5 & $1(4 \%)$ \\
\hline
\end{tabular}

graft consisting primarily of type-1 porcine collagen. It provides a scaffold and serves as a template for the constructive tissue remodelling. Experimental evidence suggests that even though the scaffold provided by the mesh rapidly degrades within the first week the remodelled tissue is much stronger than normal scar tissue. And the use of this mesh to reinforce the hiatal repair has shown to lower the recurrence of hiatus hernia [8]. However, there is still no consensus on the use of prosthetic mesh and on the preferred kind of mesh material.

The aim of this study was to evaluate and compare the side-effects with the use of porcine collagen mesh (Surgisis) in patients with and without a large hiatus hernia, and to assess whether the benefits of the surgisis mesh can be justified with the side-effects.

\section{Materials and Methods}

A retrospective and prospective review was performed of all patients from a single upper gastrointestinal surgeon's practice who underwent laparoscopic Nissen fundoplication with a 360-degree wrap and hiatoplasty. Patients between the years 2002 and 2010 were included in this study. Operative notes and patient records from the surgeons practice were analysed to collect data. All the patients had preoperative endoscopy and manometry studies. Outcome measures assessed include reflux, flatulence, dysphagia, requirement of post-op endoscopy and recurrence rates.
Dysphagia was assessed using the modified De Meester dysphagia score [12]: 1), grade 0: No dysphagia; 2), grade 1: occasional transient/intermittent sensation of food sticking; 3 ), grade 2: episodes of dysphagia requiring liquids to clear; 4), grade 3: progressive dysphagia for solids requiring medical attention; need for dilatation; bolus obstruction requiring hospital admission.

We used Ringley's experience [13], and divided the patients into four groups based on the presence of a large hiatus hernia (LHH), which includes paraoesophageal hiatus hernia and mixed hernias of size $>5 \mathrm{~cm}$ ) and the use of Surgisis mesh: LHH $(\mathrm{n}=85)$ : 1$)$ no Surgisis mesh $(\mathrm{n}=61), 2)$ with Surgisis mesh $(\mathrm{n}=24)$; No-LHH $(\mathrm{n}=117)$ : 1$)$ no Surgisis mesh $(n=91), 2)$ with Surgisis mesh $(n=26)$.

The use of the surgisis mesh in these patient groups and their post-operative side effects were compared and analysed using the Mann-Whitney non-parametric U test for continuous variables with a two-tailed P-value of 0.05 .

\section{Results}

A total of 202 patients between the years 2000 and 2010 were followed-up for a period of one year with a mean follow-up of six months. There was no significant difference in the operative time between groups. There were no intraoperative complications in either group, so all procedures were completed laparoscopically. Post-operative reflux, dysphagia, post-operative endoscopy and hernia recurrence were

Table 2. Post-Operative Side Effects in No-LHH Group

Patients without large hiatus hernia (mean 6 months follow-up)

\begin{tabular}{llllll}
\hline $\mathrm{N}=117$ & Reflux & Flatulence & Dysphagia & Post-op endoscopy & Recurrence \\
No Mesh $(\mathrm{N}=91)$ & 11 & 9 & 38 & 19 & 1 (de novo) \\
Surgisis mesh $(\mathrm{N}=26)$ & 3 & 5 & 18 & 10 & 1 (de novo) \\
\hline
\end{tabular}




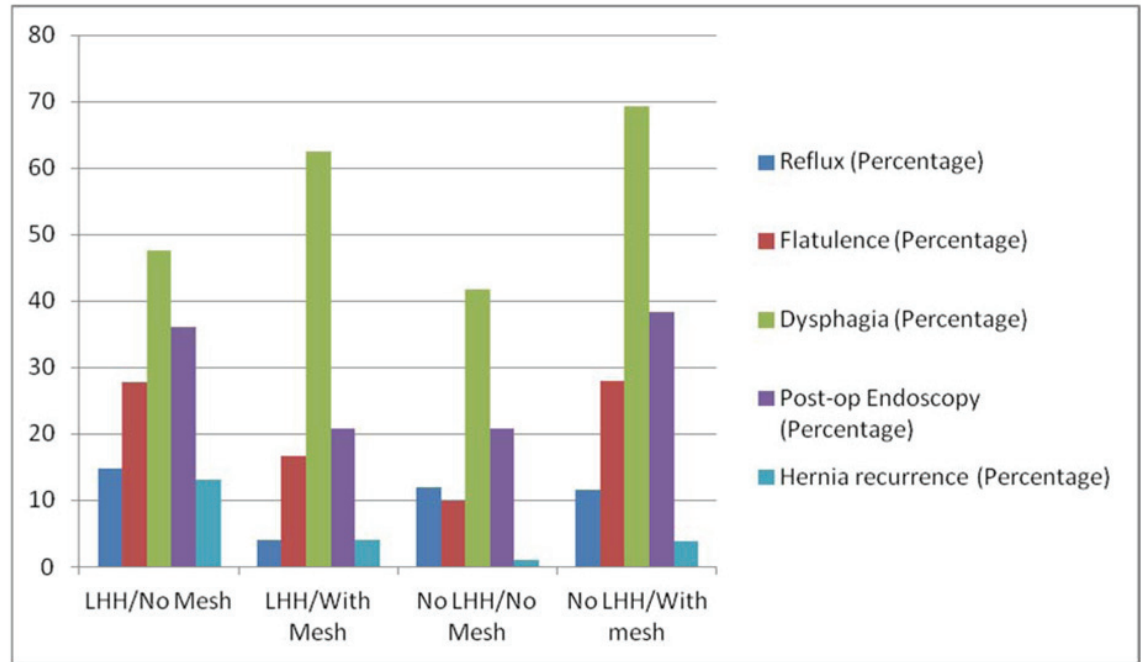

Figure 1. Percentage of patients with post-operative side-effects.

evaluated.

Patients in the mesh group had higher rate of dysphagia (62.5\% in the LHH and $69.2 \%$ in the No-LHH group). Patients in the LHH/No mesh showed a higher recurrence rate of $13 \%$ when compared to $4 \%$ in the $\mathrm{LHH} /$ mesh group. Lower recurrence rate in the surgisis group $(\mathrm{P}=0.23)$. There were 2 denovo hernia recurrences in the No-LHH group (Table 1, 2), (Fig. 1).

In the LHH/No mesh group had $15 \%$ post-operative reflux when compared to $8 \%$ in the LHH/mesh group. No significant difference in the No-LHH groups (Fig. 2).

In the LHH/No Mesh group $6.5 \%$ had preoperative dysphagia. In the LHH/with mesh group there was no dysphagia preoperatively. In No-LHH/No Mesh group 1\% had preoperative dysphagia. There were no patients with dysphagia in the No-LHH/with surgisis group (Fig. 3).

At a mean 6 months follow-up there were significant number of patients with grade- 1 dysphagia in the mesh groups $(54.1 \%$ and $53.8 \%$ in the LHH/mesh and No LHH/ mesh groups respectively when compared to $39 \%$ and $34 \%$ in the LHH/no mesh and No-LHH/no mesh groups respectively). Higher rates of grade- 3 dysphagia was reported in patients in the mesh group $(8.3 \%$ and $3.8 \%$ in the $\mathrm{LHH} / \mathrm{mesh}$ and No-LHH/mesh groups respectively when compared to $1.6 \%$ and $3.2 \%$ in the LHH/no mesh and No LHH/no mesh groups respectively) (Fig. 3).

At a mean of 12-months follow-up there was no significant difference in the rate of grade- 3 dysphagia in the LHH group, $11.4 \%$ of patients in LHH/no mesh group reported grade- 1 dysphagia when compared with $4.1 \%$ in the mesh

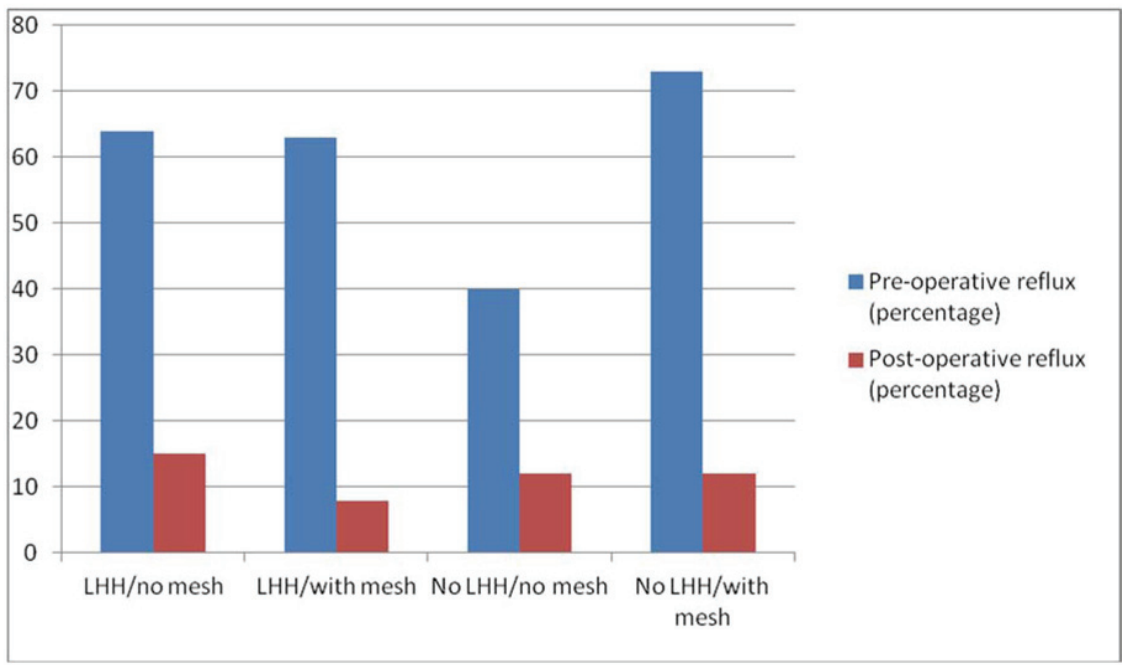

Figure 2. Percentage of patients with Pre and Postoperative Reflux. 


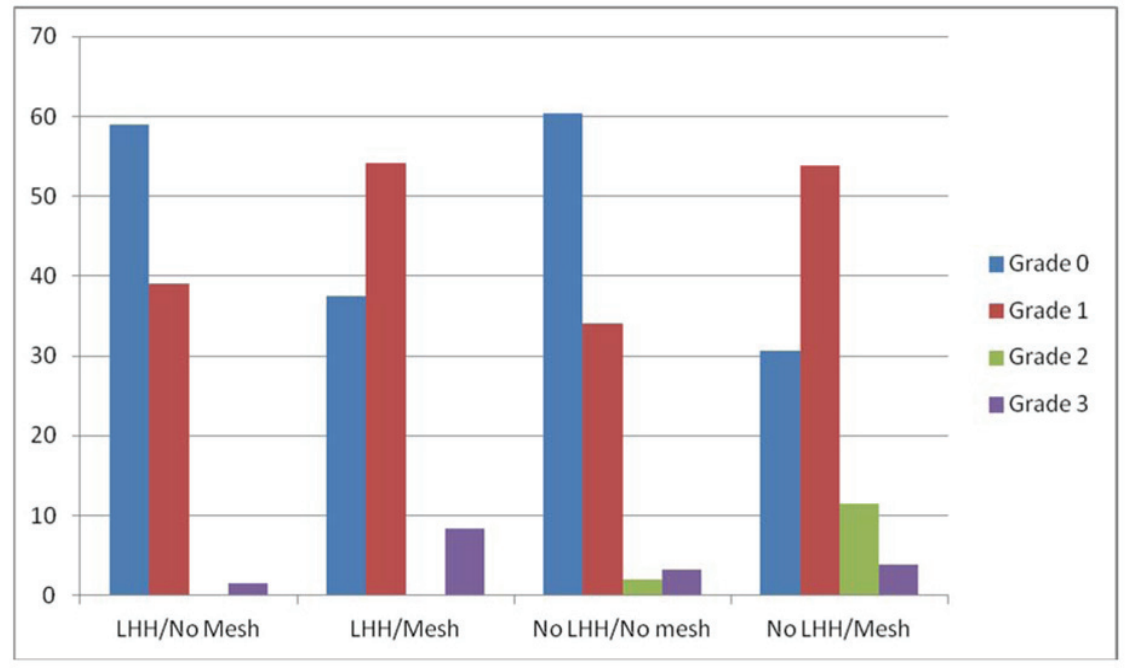

Figure 3. Percentage of patients with dysphagia at 6 months.

group. Amongst the No LHH group patients with mesh reported $23 \%$ grade- 1 dysphagia when compared to $5.4 \%$ in the No Mesh group. In patients with long-term dysphagia, we found that 5 with grade -3 dysphagia, and 3 of them requiring oesophageal dilatation (Fig. 4).

\section{Discussion}

The efficacy of laparoscopic Nissen fundoplication as an effective treatment option is exemplified by numerous studies. Many authors have shown that laparoscopic Nissen fundoplication is a safe and effective treatment option and leads to excellent functional results, relief of GORD symptoms, and a significant improvement in patient quality of life $[14,15]$.
This study shows that laparoscopic Nissen fundoplication with biological surgisis mesh repair is a safe treatment option for the management of GORD. Prospective and retrospective evaluation of reflux and dysphagia has shown relief for most patients up to one year postoperatively. Postoperative dysphagia appears to be a side effect of antireflux surgery. Majority of patients suffered from different grades of temporary dysphagia immediately after surgery. Symptomatic evaluation at 12 months showed better results for the control of reflux and dysphagia than were seen at 6 months. Overall short-term dysphagia at mean 6 months was $29 \%$ higher in the Surgisis group $(\mathrm{P}=0.001)$ and long-term dysphagia rate at 12 months was $18 \%$ higher in the Surgisis group (P $=0.015)$. Although our data present a non-randomised comparison, results support reinforcement of the hiatus with sur-

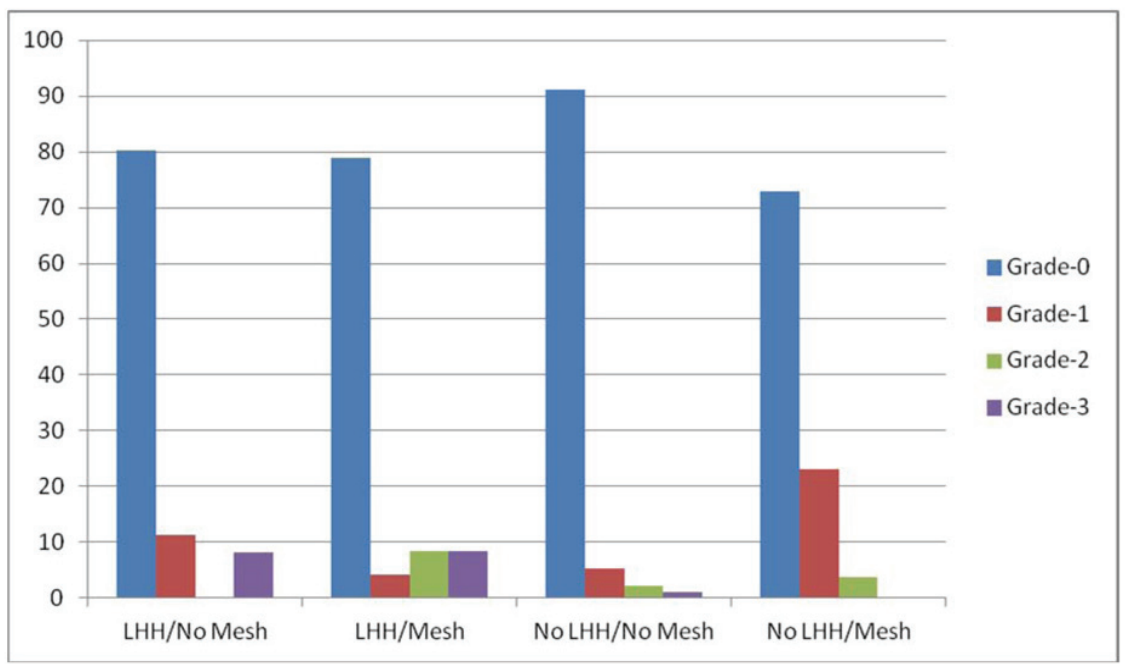

Figure 4. Percentage of patients with dysphagia at 12 months. 
gisis which leads to a reduction in hiatal-related complications. Initial post-operative dysphagia is possibly increased in intensity but only temporary.

Despite a success rate of 85 to $95 \%$ reported in large series with a mid- and long-term follow-up evaluation, important complications are related to the hiatoplasty. These complications, including intrathoracic wrap migration and hiatal hernia recurrence, result from inadequate closure of the hiatal crura or disruption of the hiatoplasty. Both anatomic and ultrastructural characteristics of the hiatal crus as well as the mean diameter of hiatal defect seem to play a key role in the development of this type of complication. Crural closure generates a lateral tension proportional to the hiatal defect diameter, which may lead to disruption of hiatal repair during inspiratory movements of the diaphragm or forceful vomiting [16]. Therefore, anything that increases the intraabdominal pressure such as lifting or falls puts strain on the diaphragm. Although prosthetic reinforcement of the crural closure seems to lower the incidence of postoperative hiatal hernia recurrence and intrathoracic wrap migration in patients who undergo prosthetic hiatal closure, still, no consensus exists concerning the routine or selective use of prosthetic mesh, its shape, size, type composition, and most of all, the long-term incidence of mesh-related complications $[14,17,18]$.

\section{Conclusion}

In conclusion, laparoscopic antireflux surgery with a biological porcine collagen surgisis mesh used to close the crura in cases of hiatal hernia is an effective and safe procedure. The use of surgisis in patients without LHH is questionable due to higher rate of early dysphagia .Most patients experience good to excellent functional and symptomatic results. Randomised controlled trials with long-term follow-up are important before we can generalise the outcomes of this study.

\section{References}

1. Cookson R, Flood C, Koo B, Mahon D, Rhodes M. Short-term cost effectiveness and long-term cost analysis comparing laparoscopic Nissen fundoplication with proton-pump inhibitor maintenance for gastro-oesophageal reflux disease. Br J Surg. 2005;92(6):700-706.

2. Buyske J. Laparoscopic nissen fundoplication. Operative techniques in general surgery. 2007; 9 (3): 95-103.

3. Lundell L, Attwood S, Ell C, Fiocca R, Galmiche JP, Hatlebakk J, Lind T, et al. Comparing laparoscopic antireflux surgery with esomeprazole in the management of patients with chronic gastro-oesophageal reflux disease: a 3-year interim analysis of the LOTUS trial. Gut. 2008;57(9):1207-1213.

4. Galmiche JP, Hatlebakk J, Attwood S, Ell C, Fiocca
R, Eklund S, Langstrom G, et al. Laparoscopic antireflux surgery vs esomeprazole treatment for chronic GERD: the LOTUS randomized clinical trial. JAMA. 2011;305(19):1969-1977.

5. Zugel N, Lang RA, Kox M, Huttl TP. Severe complication of laparoscopic mesh hiatoplasty for paraesophageal hernia. Surg Endosc. 2009;23(11):2563-2567.

6. Lundell L. Surgical therapy of gastro-oesophageal reflux disease. Best Pract Res Clin Gastroenterol. 2010;24(6):947-959.

7. Herbella FA, Patti MG, Del Grande JC. Hiatal mesh repair--current status. Surg Laparosc Endosc Percutan Tech. 2011;21(2):61-66.

8. Oelschlager BK, Barreca M, Chang L, Pellegrini CA. The use of small intestine submucosa in the repair of paraesophageal hernias: initial observations of a new technique. Am J Surg. 2003;186(1):4-8.

9. Jansen M, Otto J, Jansen PL, Anurov M, Titkova S, Willis S, Rosch R, et al. Mesh migration into the esophageal wall after mesh hiatoplasty: comparison of two alloplastic materials. Surg Endosc. 2007;21(12):2298-2303.

10. Attwood SE, Lundell L, Ell C, Galmiche JP, Hatlebakk J, Fiocca R, Lind T, et al. Standardization of surgical technique in antireflux surgery: the LOTUS Trial experience. World J Surg. 2008;32(6):995-998.

11. Tatum RP, Shalhub S, Oelschlager BK, Pellegrini CA. Complications of PTFE mesh at the diaphragmatic hiatus. J Gastrointest Surg. 2008;12(5):953-957.

12. Stadlhuber RJ, Sherif AE, Mittal SK, Fitzgibbons RJ, Jr., Michael Brunt L, Hunter JG, Demeester TR, et al. Mesh complications after prosthetic reinforcement of hiatal closure: a 28-case series. Surg Endosc. 2009;23(6):12191226.

13. Gotley DC, Smithers BM, Rhodes M, Menzies B, Branicki FJ, Nathanson L. Laparoscopic Nissen fundoplication--200 consecutive cases. Gut. 1996;38(4):487491.

14. Ringley CD, Bochkarev V, Ahmed SI, Vitamvas ML, Oleynikov D. Laparoscopic hiatal hernia repair with human acellular dermal matrix patch: our initial experience. Am J Surg. 2006;192(6):767-772.

15. Granderath FA, Schweiger UM, Kamolz T, Asche KU, Pointner R. Laparoscopic Nissen fundoplication with prosthetic hiatal closure reduces postoperative intrathoracic wrap herniation: preliminary results of a prospective randomized functional and clinical study. Arch Surg. 2005;140(1):40-48.

16. Parameswaran R, Ali A, Velmurugan S, Adjepong SE, Sigurdsson A. Laparoscopic repair of large paraesophageal hiatus hernia: quality of life and durability. Surg Endosc. 2006;20(8):1221-1224.

17. Targarona EM, Bendahan G, Balague C, Garriga J, Trias M. Mesh in the hiatus: a controversial issue. Arch Surg. 2004;139(12):1286-1296; discussion 1296. 
18. Keidar A, Szold A. Laparoscopic repair of paraesophageal hernia with selective use of mesh. Surg Laparosc Endosc Percutan Tech. 2003;13(3):149-154.
19. Soricelli E, Basso N, Genco A, Cipriano M. Long-term results of hiatal hernia mesh repair and antireflux laparoscopic surgery. Surg Endosc. 2009;23(11):2499-2504. 